\title{
DIAGNÓSTICO ENERGÉTICO: CONCEITOS E APLICAÇÕES PARA GRADUANDOS EM ENGENHARIA
}

\author{
Danieli Soaresde Oliveira-danieli@ifes.edu.br \\ IFES-ES \\ Rodovia Governador José Sette, 184 - Itacibá \\ 29.150-410 - Cariacica - ES
}

Resumo: Este artigo propõe metodologias alternativas de discussão sobre conceitos e aplicações da temática diagnóstico energético para graduandos em engenharia baseado em metodologias de aprendizagem significativa. Duas atividades são propostas: a primeira se baseia em uma análise comparativa entre empreendimentos (edificações e industriais) e o corpo humano, visando relacionar conhecimentos prévios do discente com conceitos novos, permitindo a ampliação e atualização de informações. A segunda se baseia na utilização da animação Monstros S.A., criada a partir da parceria entre a Pixar Animation Studios e a Walt Disney Pictures, como estratégia pedagógica alternativa no ensino de eficiência energética, em especial diagnóstico energético, para estudantes de engenharia. Temáticas de grande relevância para a formação em engenharia são apresentadas nessa animação; dentre elas destacam-se o aproveitamento de oportunidades em tempos de crise, identificação de riscos, inovação, produtividade, estratégia empresarial, liderança e trabalho em equipe. As metodologias alternativas de ensino-aprendizagem propostas neste artigo foram aplicadas experimentalmente em discentes do Instituto Federal do Espírito Santo, no curso de pós graduação lato sensu em Eficiência Energética no primeiro semestre de 2019. Os resultados satisfatórios obtidos indicam as potencialidades de aplicação desta metodologia em cursos de graduação em Engenharia, haja vista a importância da temática em âmbito ambiental, social e econômico.

Palavras-chave: Diagnóstico energético predial. Engenharia. Metodologias alternativas de ensino-aprendizagem. Eficiência energética.

\section{INTRODUÇÃO}

Eficiência energética tem sido um tópico bastante discutido em diversos meios de comunicação técnicos e científicos, tanto para fins industriais como prediais (AGYARKO; OPOKU; VAN BUSKIRK, 2020; BHADBHADE; PATEL, 2020; MALINAUSKAITE; JOUHARA; EGILEGOR; AL-MANSOUR et al., 2020; NURCAHYANTO; SIMSEK; URMEE, 2020; WEEBER; GHISI; SAUER, 2018). Sua evolução tem sido detalhadamente avaliada visando, essencialmente, o entendimento da diferença de desempenho entre empreendimentos e regiões, além do consumo racional de energia e a redução na emissão de poluentes (BHADBHADE; YILMAZ; ZUBERI; EICHHAMMER et al., 2020; CHENG, 2016; CZAKÓ, 2012; LIN; ZHENG, 2017; RUZZENENTI; BASOSI, 2009).

Uma ferramenta bastante utilizada para obtenção de informações acerca dos empreendimentos para avaliações de eficiência energética é o diagnóstico energético, definido como a análise sistemática do uso de energia a fim de identificar, quantificar e relatar as oportunidades para um melhor desempenho energético (ABNT, 2014). DALMOLIN (2018) 
considera o diagnóstico energético como a principal ferramenta para projetos de eficiência energética, visto que é por meio dele que se pode verificar as deficiências existentes e propor melhorias e benefícios, evitando que investimentos sejam feitos em projetos que não trarão o resultado esperado.

São verificados na literatura casos de sucesso de diagnósticos energéticos que culminaram em retornos financeiros, ambientais e sociais para as entidades envolvidas. Como exemplo, OLIVEIRA FILHO; SAMPAIO; MORAES; PIZZIOLO et al. (2011) apresentam o desenvolvimento de uma metodologia para diagnosticar energeticamente sistemas de captação de água com vistas ao abastecimento da irrigação e para meio urbano. Foram avaliadas a qualidade da energia elétrica fornecida pela concessionária; o consumo e a demanda de energia elétrica na estação de captação; as características elétricas e hidráulicas das cargas; o enquadramento e a otimização tarifárias; a capacidade de armazenamento de água e o gerenciamento dos horários de funcionamento, com um potencial de economia de energia elétrica de até $52 \%$. Já no diagnóstico energético descrito no trabalho de GARCÍA-FAJARDO; CAICEDO-CUCHIMBA; TOBAR-ESCOBAR e FLÓREZ-MARULANDA (2019), referente ao setor industrial de plásticos, foi executada uma revisão geral da organização em estudo, além da avaliação de usos de energia, identificação do potencial de economia e proposição de melhoria da eficiência energética. Uma série de ações foram propostas e uma melhoria na ordem de 7,25\% de eficiência energética foi obtida, além da otimização da produção. Em SILVA; NETO e LIRA (2018) foram verificados significativos valores de perdas em instituições públicas de ensino superior multicampi por meio de diagnósticos energéticos e análises de benchmarking ${ }^{l}$. Falhas no gerenciamento do uso de energia elétrica foram apuradas por meio da análise das faturas de consumo; com base nesses dados foram propostas estratégias para o aumento da eficiência energética, visando principalmente a sensibilização da comunidade acadêmica às questões ambientais, estimulando o desenvolvimento de espaços educacionais sustentáveis.

Nessa vertente, diversos institutos de engenharia têm incluído em seus currículos tópicos relacionados à eficiência energética; como destaque, são apresentados os trabalhos de DESHA e HARGROVES (2010) e DESHA; ROBINSON e SPROUL (2015). Em DESHA e HARGROVES (2010) são apresentados os resultados de uma pesquisa com 27 universidades australianas de engenharia, com o objetivo de verificar a extensão da educação em eficiência energética (EE) e identificar métodos para auxiliar na expansão do ensino de ações de eficiência energética nos currículos dos cursos de engenharia. $\mathrm{O}$ artigo destaca várias opções para apoiar os educadores a incorporar a sustentabilidade nos programas de engenharia e oportunidades futuras para monitorar a eficiência energética dentro do contexto da educação em engenharia para o desenvolvimento sustentável. Já em DESHA; ROBINSON e SPROUL (2015) são discutidas iniciativas bem-sucedidas em treinamentos em eficiência energética, onde os principais setores de atuação incluem construção, manufatura e serviços. Tais trabalhos indicam que o ensino de eficiência energética na engenharia, em especial análises de diagnóstico energético, são de grande relevância para os futuros profissionais em formação. No âmbito nacional, alguns cursos de engenharia, incluindo a instituição onde esta pesquisa foi desenvolvida, apresentam em seus cursos de graduação disciplinas que retratam, direta ou indiretamente, o assunto eficiência energética, o que demonstra um grande avanço no ensino brasileiro de engenharia.

\footnotetext{
${ }^{1}$ benchmarking energético consiste na comparação do consumo de energia entre diversos elementos (edifícios, equipamentos, dentre outros) de uma mesma tipologia, que servem a um mesmo propósito ou que tenham características semelhantes.
} 
Mudanças no ambiente educacional, em especial no ensino superior, são evidentes, como visto nos trabalhos de DESHA e HARGROVES (2010) e DESHA; ROBINSON e SPROUL (2015). O ambiente educacional tem sofrido constantes alterações nas últimas décadas, promovendo o surgimento de diversas iniciativas que visam apoiar e fomentar ações para a melhoria da qualidade da educação, como forma de modificar o cenário educacional, tanto nas práticas docentes quanto no desempenho dos estudantes. Estratégias inovadoras, contextualizadas e que utilizem recursos que ampliem as perspectivas da aprendizagem, podem tornar-se opções efetivas na melhoria do processo de ensino- aprendizagem (LEDUR; MOLON, 2015). Para que ocorra a aprendizagem significativa dos conteúdos ministrados são necessárias mudanças na postura de discentes e docentes; os discentes devem estar abertos para a obtenção de novos conhecimentos, enquanto que os docentes necessitam atualizar suas metodologias de ensino, optando por alternativas didáticas que favoreçam a construção da aprendizagem de forma significativa, na qual o estudante utiliza conhecimentos prévios para a construção de novos conhecimentos (SILVA; BRAIBANTE, 2018). Dessa forma, a aprendizagem se efetiva quando o aluno percebe significado nas informações recebidas (MUCH; BONFADA; TERRAZZAN, 2018).

Dada a importância dos assuntos eficiência energética e diagnóstico energético para áreas tecnológicas, em especial para engenharias, e baseando-se nos resultados exitosos obtidos em trabalhos relatados pela literatura, baseados em metodologias alternativas de ensinoaprendizagem e em aprendizagem significativa, foi definido o objetivo deste trabalho. Este trabalho propõe metodologias alternativas de discussão e consolidação sobre conceitos e aplicações da temática diagnóstico energético para graduandos em engenharia, nas suas diversas especialidades tecnológicas.

\section{MÉTODOS E RECURSOS}

Para o desenvolvimento desta pesquisa, que visa a proposição de metodologias alternativas de discussão sobre conceitos e aplicações da temática diagnóstico energético com graduandos em engenharia, são apresentadas no Quadro 1 duas propostas de atividades. Essas atividades visam aproveitar os conhecimentos prévios dos estudantes para posterior ampliação e atualização de conhecimentos, proporcionando a eles uma aprendizagem significativa acerca de eficiência energética e diagnóstico energético.

Quadro 1 - Atividades propostas para a discussão com graduandos em engenharia sobre conceitos e aplicações da temática diagnóstico energético.

\begin{tabular}{|c|l|l|}
\hline Atividade & \multicolumn{1}{|c|}{ Descrição } & \multicolumn{1}{c|}{ Objetivos } \\
\hline 1 & $\begin{array}{l}\text { Comparação entre sistemas } \\
\text { - corpo humano versus } \\
\text { empreendimentos } \\
\text { (edificações e indústrias). }\end{array}$ & $\begin{array}{l}\text { - Comparar entradas e saídas nos dois sistemas; } \\
\text { humano e os empreendimentos; }\end{array}$ \\
& & - Comparar diagnóstico médico e energético; \\
& & $\begin{array}{l}\text { - Comparar o objetivo dos diagnósticos nos dois } \\
\text { sistemas. }\end{array}$ \\
\hline
\end{tabular}




\begin{tabular}{|l|l|l|}
\hline 2 & $\begin{array}{l}\text { Uso do filme Monstros } \\
\text { S.A. para discussões acerca } \\
\text { das temáticas eficiência } \\
\text { energética e diagnóstico } \\
\text { energético. }\end{array}$ & $\begin{array}{l}\text { Criar um espaço de debate/ discussão sobre } \\
\text { eficiência energética e diagnóstico energético } \\
\text { utilizando o universo lúdico dos filmes de } \\
\text { animação. }\end{array}$ \\
\hline
\end{tabular}

Fonte: Própria autora

As atividades propostas foram previamente testadas em uma turma de pós graduação lato sensu em Eficiência Energética do Instituto Federal do Espírito Santo, na disciplina de Diagnóstico Energético Predial, lecionada pela autora deste trabalho no ano de $2019-1^{\circ}$ semestre. Essa turma foi utilizada como turma experimental para avaliação das metodologias apresentadas nesse trabalho para posterior aplicação em turmas de graduação em engenharia. Em especial, a docente pretende aplicar as metodologias apresentadas neste artigo no curso de graduação em Engenharia de Produção do Instituto Federal do Espírito Santo campus Cariacica, no qual a docente leciona a disciplina optativa "Soluções sustentáveis na indústria de bens e serviços".

Os resultados satisfatórios obtidos na turma experimental motivaram a escrita deste artigo, com possibilidade de aplicação em diversos cursos de engenharia (com as devidas adequações/ especificidades). Cada atividade foi desenvolvida para ser aplicada em aulas distintas, na ordem apresentada no Quadro 1.

\section{RESULTADOS E DISCUSSÃO}

São detalhados e discutidos nessa seção as metodologias alternativas propostas neste trabalho sobre a temática diagnóstico energético. A atividade 1 refere-se à comparação entre sistemas - corpo humano versus empreendimentos (edificações e indústrias); e a atividade 2 refere-se ao uso do filme Monstros S.A. para discussões acerca das temáticas eficiência energética e diagnóstico energético.

\subsection{Atividade 1}

A atividade 1 foi desenvolvida com a turma experimental mostrando inicialmente a semelhança entre os sistemas corpo humano e empreendimentos (edificações e indústrias): em ambos são necessários insumos (tais como água e energia) e são eliminados resíduos. Foi discutido pelos discentes os diversos sistemas que compõem o corpo humano (respiratório, circulatório, nervoso, dentre outros) e os diversos sistemas que compõem uma edificação ou complexo industrial (sistema elétrico, refrigeração, informação, dentre outros). Um paralelo entre diagnóstico médico e energético também foi traçado pelos alunos: no primeiro caso fazse um diagnóstico médico quando se tem algum sintoma físico de mal funcionamento de algum sistema que compõe o corpo humano; já o diagnóstico energético é realizado quando se tem algum sintoma de mal funcionamento do sistema de energia, como alto consumo energético ou falha constante de equipamentos elétricos. Ao final da atividade os alunos discutiram etapas e informações necessárias para a realização de diagnósticos. No caso do corpo humano, informações são levantadas por meio de análises clínicas e exames, com coleta de informações e descrição de hábitos saudáveis e não saudáveis, para posterior proposição de alternativas/ soluções. Os alunos enfatizaram que mudanças de hábitos (com retirada de hábitos nocivos exemplo: cigarro), substituições de elementos com mal funcionamento (exemplo: implantes dentários) e utilização adequada de energia (exemplo: ajuste de ingestão de água e alimentos) estão entre os pontos principais a serem avaliados em diagnósticos médicos. No caso de 
edificações e complexos industriais os alunos deduziram que, dadas as devidas diferenças e proporções, a obtenção de informações e a proposição de soluções era consideravelmente semelhante. Verificaram a importância de obtenção das características do local (área construída, tipo de ventilação e iluminação, dentre outros) por meio de análises e exames, e levantamento de hábitos de consumo (energia e água, por exemplo). Também verificaram que a proposição de alternativas e soluções era baseada, essencialmente, em mudanças de hábitos (ex.: desligar o sistema de ar condicionado ao término do expediente); substituições (ex.: substituição de sistema antigo de ar condicionado por um mais eficiente); e utilização adequada da energia (ex.: verificar se a quantidade de energia utilizada é compatível com o tipo de atividade).

Os resultados obtidos na atividade 1 superaram as expectativas da docente da disciplina, com alto interesse dos alunos na atividade proposta, com diversos momentos de debate e troca de ideias. Após a comparação entre os sistemas corpo humano e empreendimentos (edificações e indústrias), os alunos foram apresentados a norma ABNT ISO 50002 (ABNT, 2014) para discussão das etapas de um diagnóstico energético. As etapas foram apresentadas para os alunos e, com base nas discussões iniciais da aula, os discentes organizaram as etapas de um diagnóstico energético em ordem lógica/ temporal, com posterior comparação ao apresentado na norma (Figura 1). A montagem das etapas foi utilizada pela docente como ferramenta de verificação de aprendizagem. A Figura 2 apresenta o momento em que os discentes da turma experimental discutiam as etapas de um diagnóstico energético; é importante destacar que os discentes tiveram êxito no desenvolvimento do exercício proposto como verificação da aprendizagem.

Figura 1 - Fluxograma do processo de diagnóstico energético.

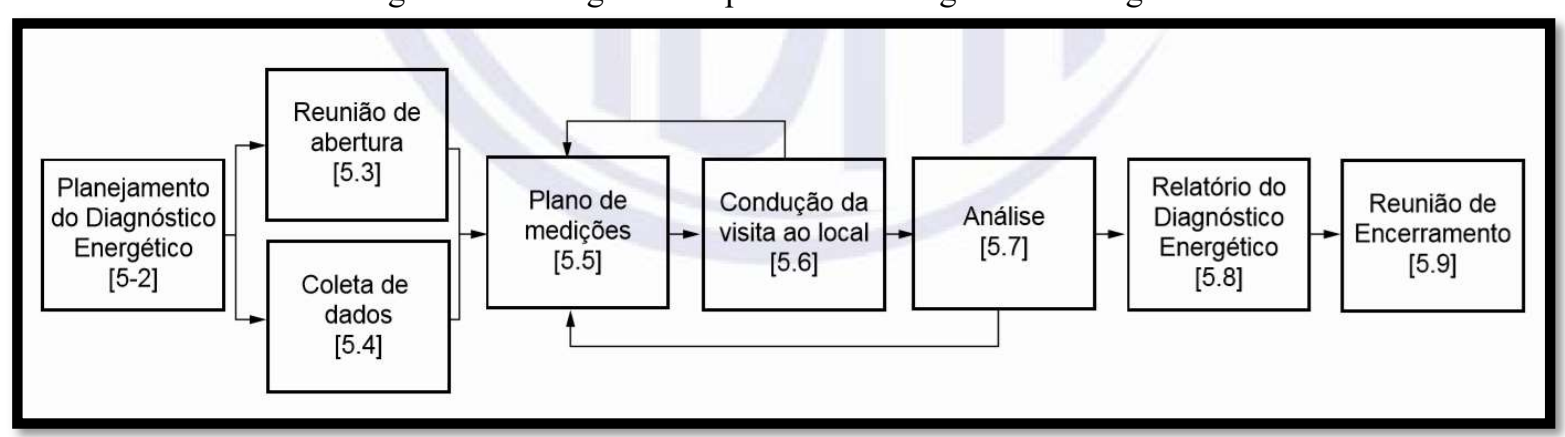

Fonte: NBR ISO 50002 
Figura 2 - Foto da turma piloto na atividade 1.

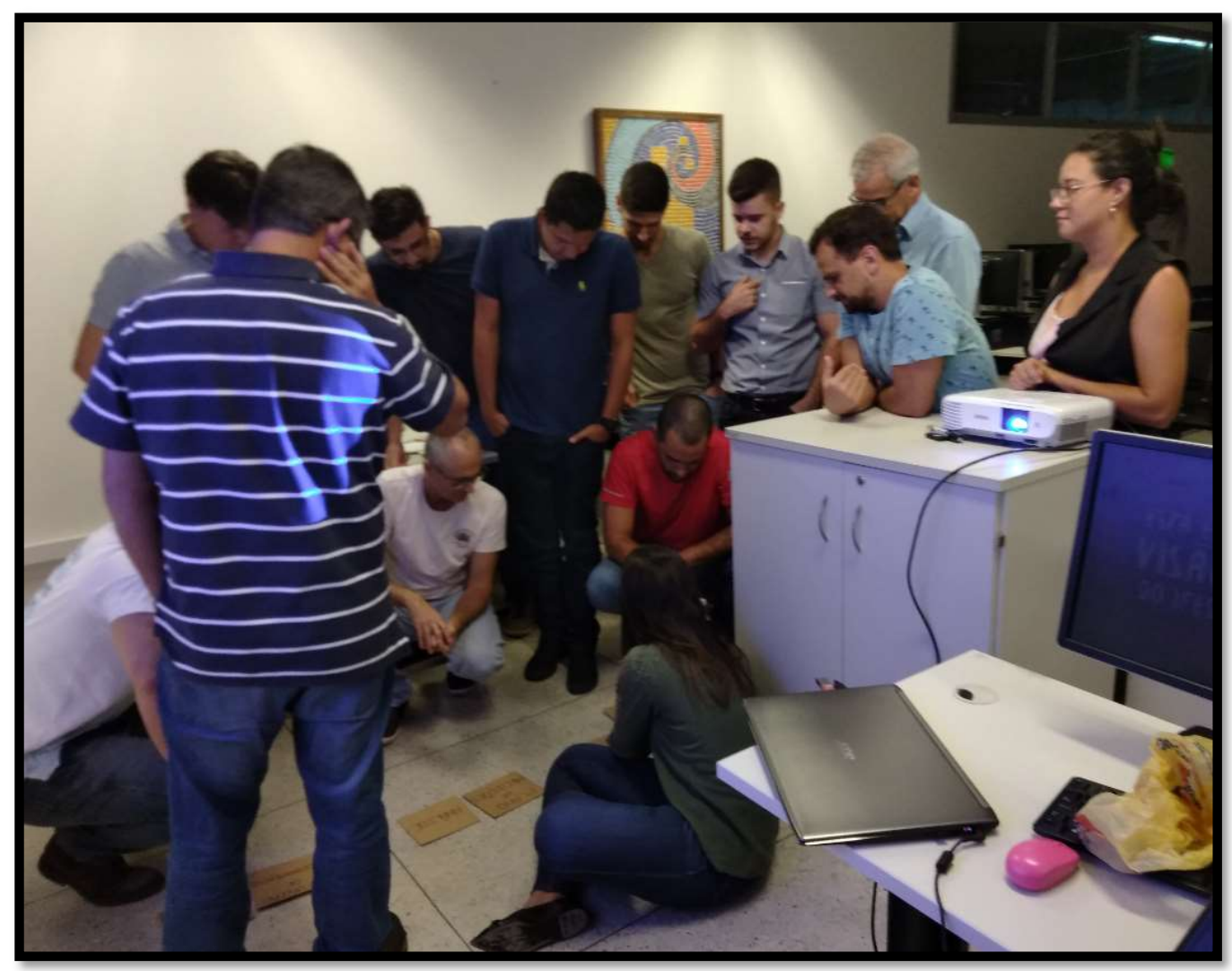

Fonte: Própria autora

\subsection{Atividade 2}

A atividade 2 foi desenvolvida com a turma experimental na forma de roda de discussão e questões iniciais norteadoras. Foi requisitado aos discentes que assistissem o filme de animação Monstros S.A. e, no encontro posterior, as questões norteadoras foram apresentadas aos alunos (Quadro 2).

\begin{tabular}{|c|l|}
\hline \multicolumn{1}{|c|}{$\begin{array}{c}\text { Quadro 2 - Questões norteadoras discutidas no desenvolvimento da atividade 2. } \\
\text { norteadoras }\end{array}$} & \multicolumn{1}{c|}{ Descrição } \\
\hline$\# 1$ & $\begin{array}{l}\text { Você acha que o filme tem a ver com o assunto da nossa disciplina? } \\
\text { Justifique. }\end{array}$ \\
\hline$\# 2$ & $\begin{array}{l}\text { Qual o tipo de empresa avaliado? Como é obtida a matéria prima para } \\
\text { obtenção da energia? }\end{array}$ \\
\hline$\# 3$ & $\begin{array}{l}\text { Como é o gestor da empresa? Você o classificaria como inovador ou } \\
\text { conservador/tradicionalista? Qual tipo você prefere "enfrentar" caso } \\
\text { você seja contratado como consultor de energia para realização de um } \\
\text { diagnóstico. Por quê? }\end{array}$ \\
\hline$\# 4$ & $\begin{array}{l}\text { Há um senso de coletividade ou de competitividade entre os } \\
\text { funcionários? Você considera um melhor que o outro? Existem }\end{array}$ \\
\hline
\end{tabular}




\begin{tabular}{|c|l|}
\hline & $\begin{array}{l}\text { situações que um é melhor que o outro? Em especial, você acha que para } \\
\text { realizar um diagnóstico energético é melhor que os membros da equipe } \\
\text { possuam senso de coletividade ou competitividade? }\end{array}$ \\
\hline$\# 5$ & $\begin{array}{l}\text { Qual a relação entre esse filme e a eficiência energética? Qual é o fato } \\
\text { mais significativo no filme que retrata a importância de observar/ avaliar } \\
\text { o processo para obtenção de melhorias? }\end{array}$ \\
\hline$\# 6$ & $\begin{array}{l}\text { Ações de diagnósticos energético são executadas durante o filme? Se } \\
\text { sim, comente. }\end{array}$ \\
\hline
\end{tabular}

Fonte: Própria autora

Os resultados obtidos com a aplicação da atividade foram satisfatórios, com grande envolvimento da turma nos momentos de discussão e debate. $\mathrm{O}$ filme apresenta temáticas de grande relevância para a formação em engenharia, tais como aproveitamento de oportunidades em tempos de crise, identificação de riscos, inovação, produtividade, estratégia empresarial, liderança, trabalho em equipe, dentre outros. A metodologia alternativa baseada no uso de filmes de animação visa tornar o aprendizado mais atraente e aumentar a motivação nos discentes, propiciando um melhor desempenho e contribuindo para a formação de profissionais mais criativos. Os resultados satisfatórios obtidos na turma experimental indicam que a metodologia proposta pode ser uma ferramenta de auxílio na implementação de conceitos e práticas de eficiência energética e diagnóstico energético em cursos de graduação em engenharia.

A única ressalva refere-se ao fato de que alguns discentes podem ter dificuldades de acesso ao filme por limitações de acesso à internet ou outras ferramentas de transmissão online de vídeos. Isso torna inviável a participação de tais alunos na atividade. Para minimizar este efeito na turma experimental, um vídeo resumido do filme (de cerca de 5 minutos) foi disponibilizado para os alunos antes da aula.

\section{CONSIDERAÇÕES FINAIS}

Este artigo apresentou a proposição de metodologias alternativas de discussão sobre conceitos e aplicações da temática diagnóstico energético para discentes de engenharia baseado em metodologias de aprendizagem significativa. Duas metodologias alternativas de ensinoaprendizagem foram apresentadas, com implementação experimental em uma turma de pós graduação lato sensu em Eficiência Energética. As atividades propostas permitiram despertar o interesse dos discentes nas temáticas eficiência energética e diagnóstico energético, com amplo espaço para trocas de ideias e debates. Os resultados satisfatórios obtidos indicam as potencialidades de aplicação desta metodologia em cursos de graduação em engenharia, haja vista a importância da temática em âmbito ambiental, social e econômico.

\section{Agradecimentos}

A autora agradece o apoio financeiro e institucional do Instituto Federal do Espírito Santo. 


\section{REFERÊNCIAS}

ABNT, N. I. Diagnósticos energéticos — Requisitos com orientação para uso 2014.

AGYARKO, K. A.; OPOKU, R.; VAN BUSKIRK, R. Removing barriers and promoting demand-side energy efficiency in households in Sub-Saharan Africa: A case study in Ghana. Energy Policy, 137, p. 111149, 2020/02/01/ 2020.

BHADBHADE, N.; PATEL, M. K. Analysis of energy efficiency improvement and carbon dioxide abatement potentials for Swiss Food and Beverage sector. Resources, Conservation and Recycling, 161, p. 104967, 2020/10/01/ 2020.

BHADBHADE, N.; YILMAZ, S.; ZUBERI, J. S.; EICHHAMMER, W. et al. The evolution of energy efficiency in Switzerland in the period 2000-2016. Energy, 191, p. 116526, 2020/01/15/ 2020.

CHENG, Y. D. Dynamic evolution of energy efficiency mechanism in China. Journal of Mechanical Engineering Research and Developments, 39, n. 2, 2016.

CZAKÓ, V. Evolution of Hungarian residential energy efficiency support programmes: road to and operation under the Green Investment Scheme. Energy Efficiency, 5, n. 2, 2012.

DALMOLIN, R. S. Eficiência energética: diagnóstico energético em uma indústria de mineração de não ferrosos. 2018. - Curso de especialização em Eficiência Energética, Universidade Tecnológica Federal do Paraná, Curitiba.

DESHA, C.; ROBINSON, D.; SPROUL, A. Working in partnership to develop engineering capability in energy efficiency. Journal of Cleaner Production, 106, p. 283-291, 2015/11/01/ 2015.

DESHA, C. J.; HARGROVES, K. Surveying the state of higher education in energy efficiency, in Australian engineering curriculum. Journal of Cleaner Production, 18, n. 7, p. 652-658, 2010/05/01/ 2010.

GARCÍA-FAJARDO, M. I.; CAICEDO-CUCHIMBA， J. M.; TOBAR-ESCOBAR, V.; FLÓREZ-MARULANDA, J. F. Energy audit proposal for industry applied to a case study in the plastics sector. Dyna, 86, n. 210, p. 345-354, 2019.

LEDUR, J. R.; MOLON, G. Aprendizagem significativa da função seno. Revista eletrônica da matemática, 1, n. 2, 2015. 
LIN, B.; ZHENG, Q. Energy efficiency evolution of China's paper industry. Journal of Cleaner Production, 140, p. 1105-1117, 2017/01/01/ 2017.

MALINAUSKAITE, J.; JOUHARA, H.; EGILEGOR, B.; AL-MANSOUR, F. et al. Energy Efficiency in the Industrial sector in the EU, Slovenia, and Spain Energy, 2020.

MUCH, L. N.; BONFADA, K. M.; TERRAZZAN, E. A. Mudança na prática docente: incentivando o protagonismo discente Revista Latino-Americana de Estudos em Cultura e Sociedade 4, n. $n^{\circ} 805,2018$.

NURCAHYANTO; SIMSEK, Y.; URMEE, T. Opportunities and challenges of energy service companies to promote energy efficiency programs in Indonesia. Energy, 205, p. 117603, 2020/08/15/2020.

OLIVEIRA FILHO, D.; SAMPAIO, R. P.; MORAES, M. J. d.; PIZZIOLO, T. d. A. et al. Metodologia de diagnóstico energético em estação de captação de água. Revista Brasileira de Engenharia Agrícola e Ambiental, 15, p. 1097-1103, 2011.

RUZZENENTI, F.; BASOSI, R. Evaluation of the energy efficiency evolution in the European road freight transport sector. Energy Policy, 37, n. 10, p. 4079-4085, 2009/10/01/2009.

SILVA, J. A. S.; BRAIBANTE, M. E. F. Aprendizagem significativa: concepções na formação inicial de professores de Ciências. Revista insignare scientia, 1, n. 1, 2018.

SILVA, O. A. V. d. O. L. d.; NETO, J. M. M.; LIRA, M. A. T. Análise envoltória de dados para a gestão energética em instituições de ensino superior multicampi Revista Brasileira De Ciências Ambientais (Online), 50, p. 78-96, 2018.

WEEBER, M.; GHISI, E.; SAUER, A. Applying Energy Building Simulation in the Assessment of Energy Efficiency Measures in Factories. Procedia CIRP, 69, p. 336-341, 2018/01/01/ 2018 .

\section{ENERGY AUDITS: CONCEPTS AND APPLICATIONS FOR ENGINEERING STUDENTS}

Abstract: This article discusses the importance of the energy audits for engineering students, aiming to highlight its applicability and importance in several technological areas. In addition, two alternative teaching methods are proposed: the first is based on a comparative analysis between enterprises (buildings and industrials) and the human body, aiming to relate the student's previous knowledge with new concepts, updating the previous information promoting a meaningful learning. The second methodology is based on the use of the animation Monsters, 
INC., created by Pixar Animation Studios and Walt Disney Pictures, as an alternative pedagogical strategy for teaching energy efficiency for engineering students, especially energy audits. Relevant topics for engineering education are presented in this animation, as the use of opportunities in times of crisis, risk identification, innovation, productivity, business strategy, leadership and teamwork. The alternative teaching-learning methodologies proposed in this article were experimentally applied in postgraduate students of the Federal Institute of Espirito Santo, in Energy Efficiency course, in the first semester of 2019. Satisfactory results indicate the potentialities of applying this methodology in engineering courses, given the importance of the theme in environmental, social and economic spheres.

Keywords: Energy audits. Engineering. Alternative teaching-learning methodologies. Energy efficiency. 